\title{
A CASE OF CHOLECYSTENTEROSTOMY.
}

$\mathbf{B Y}$

\author{
A. IV. MAYO ROBSON, F.R.C.S., \\ HON. SURGLON LEEUS GENERAL INFIRMARY; LECTURER ON PRACTICAL \\ SURGERY AT THE YORKSHIRE COLLEGE; AND EXAMINER \\ IN THE VICTORIA UNIVERSITY.
}

Received September 21st-Read November 26 th, 1889.

For the notes from which the history of this case has been abstracted I am indebted to my house surgeon, Mr. F. Hudson.

Mrs. V. B-, rt. 42, was admitted into the Leeds General Infirmary January 9th, 1888, on account of a tumour on the right side of the abdomen, which was accompanied by symptoms of acute peritonitis. She had had abdominal section performed a year previously by me for pelvic discase of several years' standing, which had produced confirmed invalidism, and after the removal of the cause, a right pyosalpinx, she had been able to resume her work, and for several months had enjoyed excellent health, and been able to perform very arduous duties as a general servant in a large family, her menstrual functions being regular and painless. Three weeks before admission she began to experience pain in the right side of the abdomen without apparent cause ; she then noticed a small rounded swelling, tender on pressure, in the right hypochondriac region; there was no jaundice, and the bowels 
were regular. The swelling and pain increased, and compelled her to give up her work. She was seen by her medical man, Mr. Loe, three days before admission, when there was very marked tenderness over the swelling, which seemed to be decidedly increasing. Her general condition became rapidly worse, and nothing could be retained on the stomach, the vomit being of a dark brown colour. She suffered from great pain and intense thirst, the abdomen being tympanitic and the pulse frequent and weak.

On admission to the infirmary on January 9th the patient had an anxious expression, and lay on her back with the knees drawn up. She complained of greàt pain in the right side of the abdomen, markedly increased by pressure, deep respiration, or turning on her side. There was a distinct sense of resistance in the right hypochondriac and iliac regions, with dulness on percussion; but on account of the extreme tenderness, palpation was rendered difficult. Respirations 30 , pulse 130 .

The fæces had a normal colour. The urine was normal except that it gave Gmelin's reaction for bile-pigment.

On January 13th, 1888, she became jaundiced. It now became evident that she would soon die unless relieved by operation, and on January 14th abdominal section was performed through the upper part of the right linea semilunaris, exposing a large cyst with thickened walls, which yielded by aspiration eight ounces of foetid pus. After the gall-bladder had been emptied it was incised, and then explored, sponges having been previously packed round it. The finger was passed along the peritoneal surface of the cystic duct as far as possible, and beyond this, but inside the duct, was passed a long metal probe, this exploring as far as the junction of the cystic with the hepatic duct; the finger was also passed along the outside of the common duct as far as the duodenum, but no gallstone or other obstruction could be felt. Around the common duct, as well as over the cystic duct, plastic lymph had been thrown out; and this probably explains the subsequent course of events. 
The gall-bladder was stitched to the skin, and a drainagetube inserted, the remainder of the wound being closed by silk sutures passed through all the layers of the abdominal wall, including the peritoneum.

For the first twenty-four hours the discharge remained clear, colourless, and mucoid ; in the second twenty-four hours it became slightly tinged with bile, and on the third day the discharge appeared to be pure bile. On the fourth day a smaller drainage-tube was inserted, and the stitches were removed on the seventh. The jaundice had quite disappeared forty-eight hours after the operation. The patient made an uninterrupted recovery with the exception of having a biliary fistula, through which apparently the whole of the bile was discharged ; for both the fæces and the urine showed no trace of biliary matter, either by inspection or on chemical examination.

During the fifteen months subsequent to the operation the patient's digestion was unimpaired unless she took too much fatty matter, and then she became sickly and lost her appetite, and rather more fat than normal was passed in the motions; the bowels were quite regular without the use of aperients, and the odour was in no wise different from that of healthy fæces. Repeated measurements were made of the whole of the bile discharged during twenty-four hours, and a careful analysis of the bile thus collected was made.

The details of these and other observations will be con. sidered in a separate paper, and may, I think, have an important bearing on the physiology of the bile, as may also observations made on the action of certain drugs on the biliary secretion have a bearing on biliary therapeutics. ${ }^{1}$

Now, although the patient was in good health, her condition was a very miserable one, since no apparatus could be made to fit sufficiently accurately to catch the whole of the bile, except when she was in bed. When

1 Paper read before Royal Society (London), April 24th, 1890, and published in the 'Proceedings' of the Royal Society for 1890. 
out of bed she had to catch the overflowing bile in absorbent cotton, which was retained in position by means of a bandage, thus necessitating her frequently changing her dressings and clothes. On one occasion, when she was unable to change the wool, the wearing of her bile-saturated garments gave her a severe chill, which resulted in an attack of pelvic cellulitis.

She was so miserable at the prospect of having to go through life with her fistula, that when I mentioned to her the possibility of again turning the bile into the bowel, she said she would risk anything to be rid of her trouble.

I asked my colleagues to see her with me, and they agreed that cholecystenterostomy was perfectly justifiable if its risks were fully explained to the patient.

Her consent was at once granted, and on March 2nd, 1889, I opened the abdomen in the right linea semilunaris through the old scar, in the centre of which was the fistula, prolonging the opening two inches beyond the lower end of the cicatrix. The gall-bladder was detached from the parietes, and found to be much contracted and thickened. There was so much matting of the viscera that it was found impracticable to bring up and fix the duodenum or jejunum to the gall-bladder as at first intended; hence the hepatic flexure of the colon, lying near, was raised and encircled by an elastic ligature, after its contents had been squeezed upwards and downwards. Convenient spots having been selected on the gall-bladder and colon, a circle the size of a florin was marked by a scalpel on each viscus. Along these lines, sutures of fine chromicised catgut were passed, about an eighth of an inch apart, by means of curved sewing-needles, but these were not tightened until openings a third of an inch in diameter had been made in the centre of the circles, quite through all the coats of the two viscera concerned, and the cut edges of the mucous membrane of the colon had been sutured by a number of interrupted stitches of fine catgut to the edge of the mucous membrane of the gallbladder. The closed blades of a pair of Spencer Wells' 
pressure forceps were passed through the opening from the gall-bladder into the bowel, in order to see that it was thoroughly patent after the ligatures had been tightened. The outer row of ligatures, only involving the serous and muscular coats, were tied and cut off short.

The refreshed edges of the old fistula were then brought together by means of a continuous catgut suture, the serous surface being tucked in and a number of Lembert's sutures being further applied over the line of union.

The elastic ligature was removed from the bowel, and the circulation became immediately re-established. The sponges which had been packed below and around the colon and gall-bladder had prevented soiling of the peritoneum.

A glass drainage-tube was placed in the right kidney pouch, and brought out at the lower end of the wound in order to guard against any accident of sutures giving way.

Lastly, silk sutures were employed to bring together the parietal incision in the usual manner. The patient had a little pain, but no sickness or distention.

On the night of March 3rd a tinge of bile appeared on the dressings, showing that the over-tense sutures on the outer surface of the gall-bladder had given way, but, thanks to the drainage-tube, without any dangerous result.

On the following day the bile came freely through the drainage-tube, and on March 5th fæcal matter made its appearance mixed with bile, after which, up to the 18th, fæces and bile continued to be discharged, and then bile alone, the wound granulating and ultimately completely closing on May 6 th, when the motions were noticed to have fally regained their normal colour.

The patient, who was sent to a Convalescent Home, rapidly gained strength and weight, and reported herself in July as in perfect health. When she left the infirmary she. weighed 8 st. $4 \frac{1}{2}$ lbs., and in July her weight was 9 st. $6 \frac{1}{2} \mathrm{lbs}$.

She was shown to the members of the British Medical VOL. LXXIII. 
Association in Leeds in August, and then said that she had never been in better health.

During the time the fistula was open the menstrual functions were in abeyance. After its closure the menses returned, and have continued to recur regularly.

While the bile was being discharged externally Mrs. B - had a dislike to fat, to meat, and to sweet food, and a craving for acids such as lemons and pickles.

The operation of cholecystenterostomy was first performed by Winiwarter, who suggested its application in cases of irremediable obstruction in the common bile-duct. It has since been performed by Monastyrki, Kappeler, Socin, and Bardenheuer, but hitherto it has not been done in England. I think it has never previously been performed for biliary fistula.

The fact of the patient having within the space of four years undergone three abdominal sections is, perhaps, almost unique. Her first operation, the removal of a pyosalpinx, restored her to health and comfort after several years of distress and incapacity, besides relieving her from the constant danger of suppurative peritonitis. The second operation, cholecystotomy, for empyema of the gall-bladder, undertaken when she was apparently dying of peritonitis, undoubtedly saved her life. The third operation, cholecystenterostomy, performed for a condition which rendered her life wretched, has restored her to a condition of absolutely perfect health, for she is now strong, well, and healthy in every respect.

Numerous interesting questions arise in the case.

1st. On the benefit derived from removing diseased uterine appendages; and in this case only the diseased one was removed, the apparently sound one being retained, the menstrual functions being afterwards continued regularly and painlessly, except during the time the biliary fistula was open, when there was amenorrhœa.

2 nd. On the advisability of operating during acute peritonitis in order to find out the cause, and, if possible, rewove it. 
3rd. On the treatment of distended gall-bladder by cholecystotomy, and not by cholecystectomy.

4th. On the cause of empyema of the gall-bladder.

5th. On the frequency or otherwise of fistula after cholecystotomy.

6th. On the apparent harmlessness to the system of the loss of the whole of the bile over so long a period as fifteen months, making it appear as if the bile were simply an excretion.

7th. The physiological experiment to which the patient voluntarily submitted herself, which will be discussed in another paper.

I would draw attention to some of the details in the operation described.

The cause of the fistula was apparently a cicatricial contraction of the duct. It was, therefore, hopeless to attempt to secure a return of the bile to the intestine by the ordinary channel, and on opening the abdomen it was found impossible, on account of the old adhesions, to stitch the gall-bladder to the small intestine in the region of the duodenum. It was therefore sutured to the colon. Instead of using the ordinary intestinal clamps, or passing ligatures through the mesentery and around the bowel, a loop of colon, after its contents had been squeezed out, was simply drawn up and secured at its base by an ordinary piece of elastic drainage-tube, which was fixed by a pair of pressure forceps. This tourniquet both prevented any escape of gas or fæcal matter, and rendered the intestine almost bloodless-in fact, it simplified the operation very considerably, and was applied in a few seconds; hence in future I shall never think of using any other intestinal clamp.

There is nothing calling for mention in the mode of application of the sutures, which were applied after the Czerny-Lembert method.

The loss of tissue from the outer surface of the gallbladder, where it had been stitched to the skin for solong a period, rendered it necessary to apply more teusion than 
was desirable in order to secure exact apposition of serous surfaces. I therefore thought it wise to insert a glass drainage-tube into the right kidney pouch in case of the escape of any bile or fæces. This precaution prevented a catastrophe when the tense sutures gave way.

As I assumed that the escape came from the outer surface of the gall-bladder, and not from its junction with the intestine, I felt confident that the fistula would ultimately close by granulation, and that then the bile would be able to flow through the new channel. I am glad to say that this prognosis was justified by the course of events.

I must apologise for giving the history at some length, but $I$ hope the interesting questions raised by this somewhat unique case may afford a sufficient excuse.

(For report of the discussion on this paper, see 'Proceedings of the Royal Medical and Chirurgical Society,' Third Series, vol. ii, p. 28.) 